\title{
Analytical methods of antibody surface coverage and orientation on bio-functionalized magnetic beads: application to immunocapture of TNF- $a$
}

\author{
Etienne Laborie $^{1,2} \cdot$ Victor Le-Minh $^{1} \cdot$ Thanh Duc Mai $^{1} \cdot$ Mehdi Ammar $^{2} \cdot$ Myriam Taverna $^{1,3} \cdot$ Claire Smadja $^{1}$
}

Received: 4 May 2021 / Revised: 3 August 2021 / Accepted: 6 August 2021 / Published online: 17 August 2021

(C) Springer-Verlag GmbH Germany, part of Springer Nature 2021

\begin{abstract}
The use of magnetic beads bio-functionalized by antibodies $(\mathrm{Ab})$ is constantly increasing with a wide range of biomedical applications. However, despite an urgent need for current methods to monitor Ab's grafting process and orientation, existing methods are still either cumbersome and/or limited. In this work, we propose a new simple and rapid analytical approach to evaluate antibody orientation and density on magnetic beads. This approach relies on the cleavage by IdeS, a highly specific protease for human immunoglobulin $\mathrm{G}$ (hIgG), of immobilized antibodies. The $\mathrm{F}(\mathrm{ab})_{2}$ and $\mathrm{Fc}$ fragments could be then accurately quantified by size exclusion chromatography (SEC)-coupled to fluorescent detection (FLD), and the ratio of these fragments was used to give insight on the IgG orientation at the bead surface. Four different commercially available magnetic beads, bearing carboxyl groups, tosyl groups, streptavidin, or protein $\mathrm{G}$ on their surface have been used in this study. Results obtained showed that this approach ensures reliable information on hIgG orientation and bead surface coverage. Protein G magnetic beads demonstrated an optimal orientation of antibodies for antigen capture ( $75 \%$ of accessible $\mathrm{F}(\mathrm{ab})_{2}$ fragment) compared to tosylactivated, carboxylated, and streptavidin ones. Capture efficiency of the different functionalized beads towards human TNF- $\alpha$ immunocapture, a biomarker of inflammation, has been also compared. Protein $G$ beads provided a more efficient capture compared to other beads. In the future, this approach could be applied to any type of surface and beads to assess hIgG coverage and orientation after any type of immobilization.
\end{abstract}

Keywords Magnetic beads, antibody $\cdot$ Orientation, immunocapture $\cdot$ TNF- $\alpha \cdot$ Immunoassays

\section{Introduction}

Magnetic beads with their several advantages have been found to have increasing applications in many fields including drug discovery, biomedicine, bioassays, diagnostics, genomics, and proteomics [1,2]. A typical application is peptide/protein selective capture via magnetic separation using magnetic beads functionalized with a bio-receptor. Many recently developed

Claire Smadja

claire.smadja@universite-paris-saclay.fr

1 Université Paris-Saclay, CNRS, Institut Galien Paris-Saclay, 92296 Châtenay-Malabry, France

2 Université Paris-Saclay, CNRS, Centre de Nanosciences et de Nanotechnologies, 91120 Palaiseau, France

3 Institut Universitaire de France, Paris, France diagnostic tools (e.g., lab-on-a-chip and biosensors) are based on bio-functionalized magnetic particles with antibodies [3]. For these purposes, several types of magnetic beads are currently available differing by their sizes, shape, surface chemistry, and immobilization strategy that are crucial for antibody grafting density and orientation. Preparation of magnetic nanoparticles with a stable surface remains of paramount importance. After the coating step, bioreceptors such as antibodies will biofunctionalize the nanoparticles to target specific biomarkers for instance. A high antibody grafting density and a good orientation, which requires exposed and fully available $\mathrm{F}(\mathrm{ab})_{2}$ fragments on bead surface, are highly desirable to ensure high loading/capture of target analytes during the immuno-precipitation/enrichment or bioassay processes.

The monitoring of surface antibody density and orientation is therefore critical not only for capture or recognition efficiency but also to produce batches of functionalized magnetic beads of equal performance and quality. 
Conventional methods to assess the antibody immobilization on beads are based either on spectrophotometric or biophysical methods. Colorimetric assays (e.g., Lowry, Bradford, and BCA assays) have been shown to provide only indirect determination of protein grafting efficiency as they are mainly based on the determination of non-immobilized proteins present in the supernatant $[4,5]$. In addition, they do not provide any information on antibody orientation. Besides, many biases have been reported with these methods mainly related to possible background interferences due to nanoparticles themselves [6-8]. Atomic force microscopy, surface plasmon resonance (SPR), spectroscopic ellipsometry, and dual polarization interferometry [5,9-12] can provide indirect insight on antibody orientation mainly by measuring antibody dimensions/thickness on surfaces or shift angle. Recently, Lämmerhofer et al. proposed an approach for quantification of the protein surface coverage on gold nanoparticles using high-performance liquid chromatography (HPLC) $[4$, 13]. In this work, pepsin conjugated to gold nanoparticles is hydrolyzed $(6 \mathrm{~N} \mathrm{HCl})$. The released amino acids are tagged with a fluorescent dye and then analyzed by HPLC with fluorescence detection. The derivatized glycine measurement is used to estimate the pepsin surface coverage on nanoparticles. Kozlowski et al. gave an estimate of surface coverage of dihydrofolate reductase (DHFR)-gold particle bioconjugates, using a combination of several techniques including SDS-PAGE, UV-vis spectrophotometry, dynamic light scattering, and a fluorescencebased method [6]. These methodologies allowed a fine protein surface coverage characterization but required several steps and a combination of several techniques. In another study relying on streptavidin-magnetic beads, Gagey-Eilstein et al. communicated a chemiluminescent test to monitor the antibody grafting rate [14] and sandwich-type immunoassay using magnetic beads grafted with antibodies have been also reported $[15,16]$. All these methods, however, did not provide information on the antibody grafting orientation nor on the functionality and ability of antibodies to capture the biological target.

Herein, we report the development of a method for quantification of antibody surface coverage and monitoring of antibody grafting orientation on magnetic beads functionalized with different functional groups or chemistries. Together with a recent publication by Shen et al. [17], our study is one of the two pioneering works which shed light on both orientation and density of antibodies decorated on magnetic beads using chromatographic approaches. Shen et al. proposed a method which used a proteolysis performed by trypsin-decorated magnetic beads to quantify the number of antibodies attached to magnetic beads. LC-MS/MS of the released tryptic peptides allowed for the quantification of representative peptides of either the $\mathrm{F}(\mathrm{ab})_{2}$ or $\mathrm{Fc}$ region of the antibody. However, it is important to note that the release of $\mathrm{F}(\mathrm{ab})_{2}$ peptides from antibodies could arise from antibodies attached via one Fab fragment leading thereby to an overestimation of the number of well oriented antibodies.
Our work relies on the use of a selective proteolysis of $\mathrm{F}(\mathrm{ab})_{2}$ regions of antibodies, using $I d e S$ that cleaves antibodies with a unique degree of specificity in the hinge region leading to the release of $\mathrm{Fc}$ and $\mathrm{F}(\mathrm{ab})_{2}$ fragments [18]. Our straightforward analytical approach relied on size exclusion liquid chromatography coupled to fluorescent detection (FLD) allowing $\mathrm{F}(\mathrm{ab})_{2}$ and Fc separation according to their molecular weight (100 and $50 \mathrm{kDa}$, respectively) [17]. $\mathrm{F}(\mathrm{ab})_{2}$ and $\mathrm{Fc}$ will be clearly identified with this new approach. In our case, the quantification of the ratio $\mathrm{F}(\mathrm{ab})_{2} / \mathrm{Fc}$ is expected to be directly related to the orientation of immobilized antibodies. Indeed, after IdeS digestion, if the antibodies are grafted through their $\mathrm{Fc}$ fragment, $\mathrm{F}(\mathrm{ab})_{2}$ is released whereas when they are immobilized via Fab fragment, Fc fragment is released. The relevance and utility of the developed approach was demonstrated with a high diagnostic value application, by quantifying the human Tumor necrosis factor alpha (TNF- $\alpha$ ), a pro-inflammatory cytokine, serving for screening of major depressive disorders (MDD) and which is involved in many inflammatory disorders such as cytokine release syndrome observed in COVID 19 [19-25].

\section{Experimental}

\section{Chemicals and reagents}

IdeS FabRICATOR enzyme (5000 units) was purchased from Genovis AB (Lund, Sweden). HPLC-grade propan-2-ol and sodium di-hydrogen phosphate (NaH2PO4) were obtained from Fisher Scientific (Karlsruhe, Germany). Sodium chloride was provided from Euromedex (Souffelweyersheim, France). Thirty-two percent sodium hydroxide was purchased from VWR SAS (Fontenay-sous-Bois, France). Eighty-five percent phosphoric acid was obtained from Carlo Erba Reagents (Valde-Reuil, France). EZ-Link Sulfo-NHS-LC-biotin kit and Dynabeads MyOne Carboxylated, Tosylactivated, Streptavidin T1, Pierce Protein G $(1 \mu \mathrm{m})$ magnetic beads, and TNF alpha Human Uncoated ELISA Kit were purchased from Life Technologies SAS (Villebon-sur-Yvette, France). Human IgG from whole serum $(10.2 \mathrm{mg} / \mathrm{mL})$ and Amicon Ultra $0.5 \mathrm{~mL}$ $50-\mathrm{kDa}$ and $100-\mathrm{kDa}$ filters for ultrafiltration were provided by Merck Millipore (Darmstadt, Germany). Phosphate-buffered saline PBS $(10 \times)$, Tris-buffered saline TBS $(10 \times)$, boric acid, Tween 20, ammonium sulfate, $\mathrm{N}$-(3-dimethylaminopropyl)-N'ethylcarbodiimide hydrochloride (EDC), and Nhydroxysulfosuccinimide sodium salt (Sulfo-NHS) were purchased from Sigma-Aldrich (St. Louis, MO, USA).

\section{Apparatus and material}

Size exclusion liquid chromatography experiments were performed using an Agilent 1260 UHPLC-1260 FLD detector (Xenon lamp) (Agilent Technologies, Santa Clara, CA, 
USA). An Agilent Bio SEC-3 column ( $3 \mu \mathrm{m}$ particle size; $300 \AA$ pore structure; $300 \mathrm{~mm}$ length; $4.6 \mathrm{~mm}$ i.d.) was employed at temperature of $25^{\circ} \mathrm{C}$. The mobile phase (50 $\mathrm{mM}$ sodium phosphate, $150 \mathrm{mM} \mathrm{NaCl}$ and $10 \%$ isopropanol at $\mathrm{pH}$ 6.5) was isocratically pumped at $300 \mu \mathrm{L} /$ min. Fluorescence detection (FLD) was performed at $\lambda_{\mathrm{ex}}=$ $280 \mathrm{~nm}$ and $\lambda_{\mathrm{em}}=340 \mathrm{~nm}$. Data acquisition and instrument control were performed by Chemstation (Agilent).

\section{Methods}

\section{Preparation of bio-functionalized magnetic beads}

Model human IgG (hIgG) were grafted on beads following commercial protocols for streptavidin and protein $\mathrm{G}$ beads. For beads tosylactivated (Ts) and $\mathrm{COOH}$, the protocols have been adapted from the supplier ones. IgG concentration in the grafting solution was kept constant at $0.1 \mathrm{mg} / \mathrm{mL}$ for accurate comparison of bead grafting efficiency.

Carboxylated magnetic beads One hundred microliters of Dynabeads MyOne Carboxylated $(10 \mathrm{mg} / \mathrm{mL})$ was added to 4 LoBind Eppendorf vials of $1.5 \mathrm{~mL}$ and washed with $1 \mathrm{~mL}$ of $20 \mathrm{mM} \mathrm{NaOH}$ overnight on a rotating wheel (Tube rotator SB3, Stuart, UK) at $20 \mathrm{rpm}$ and $4{ }^{\circ} \mathrm{C}$. Beads were then concentrated using a magnet and washed 3 times with $500 \mu \mathrm{L}$ of PBS. Then, beads were resuspended in $120 \mu \mathrm{L}$ of human IgG solution at $0.66 \mathrm{mg} / \mathrm{mL}$ in PBS. Two hundred microliters of EDC $(10 \mathrm{mg} / \mathrm{mL})$ and $200 \mu \mathrm{L}$ of Sulfo-NHS $(10 \mathrm{mg} / \mathrm{mL})$ in PBS were added to the bead suspension. Finally, volume was adjusted to $800 \mu \mathrm{L}$ with PBS, reaching a final concentration of $0.1 \mathrm{mg} / \mathrm{mL}$ of $\mathrm{IgG}$ for $1 \mathrm{mg}$ of beads. Vials were then placed on a rotating wheel at $20 \mathrm{rpm}$ overnight at $4{ }^{\circ} \mathrm{C}$ for antibody grafting, and beads were immobilized on a magnet and washed 2 times with $500 \mu \mathrm{L}$ of a solution of PBS, Tween $20(0.05 \%)$ (PBS-Tween), and one time with $500 \mu \mathrm{L}$ of PBS. Washing solutions were kept at $4{ }^{\circ} \mathrm{C}$ until analysis.

Tosylactivated magnetic beads Ten microliters of Dynabeads MyOne Tosylactivated $(100 \mathrm{mg} / \mathrm{mL})$ was added to 4 LoBind Eppendorf vials of $1.5 \mathrm{~mL}$ and washed with $500 \mu \mathrm{L}$ of $0.1 \mathrm{M}$ sodium borate buffer $\mathrm{pH} 9.5$, gently vortexed, and stirred at the same time using an Eppendorf Thermomixer for $15 \mathrm{~min} \mathrm{C}$ at $25{ }^{\circ} \mathrm{C}$ and $650 \mathrm{rpm}$. Beads were resuspended in $60 \mu \mathrm{L}$ of hIgG solution at $0.66 \mathrm{mg} / \mathrm{mL}$ in PBS with $60 \mu \mathrm{L}$ of $3 \mathrm{M}$ ammonium borate buffer $\mathrm{pH} 9.5$ and $20 \mu \mathrm{L}$ of $0.1 \mathrm{M}$ sodium borate buffer. Vials containing beads were then incubated at $37^{\circ} \mathrm{C}$ and stirred at $650 \mathrm{rpm}$ on a Thermomixer for $16 \mathrm{~h}$ and then sonicated for $5 \mathrm{~min}$ to prevent their adsorption on the vial's wall. Beads were immobilized on a magnet and washed 2 times with $500 \mu \mathrm{L}$ of a solution of PBS-Tween, and one time with $500 \mu \mathrm{L}$ of PBS. Washing solutions were kept at $4{ }^{\circ} \mathrm{C}$ until their analysis.
Streptavidin magnetic beads Biotinylation of human IgG was done using EZ-link sulfo-NHS-LC-biotin kit. Ten millimolars of solution of biotin was prepared by adding $224 \mu \mathrm{L}$ of ultrapure water to $1 \mathrm{mg}$ of biotin. $6.66 \mu \mathrm{L}$ of biotin solution was added to $1000 \mu \mathrm{L}$ of hIgG solution $(0.5 \mathrm{mg} / \mathrm{mL})$ and incubated at $25{ }^{\circ} \mathrm{C}$ for $30 \mathrm{~min}$ and stirred at $300 \mathrm{rpm}$ on a Thermomixer at the same time. The solution was then ultrafiltrated on a $50-\mathrm{kDa}$ filter cap at $4{ }^{\circ} \mathrm{C}, 10,000 \mathrm{rpm}$ for $4 \mathrm{~min}$ to get rid of biotin salts excess.

One hundred microliters of Dynabeads MyOne Streptavidin T1 $(10 \mathrm{mg} / \mathrm{mL})$ is added to 4 LoBind Eppendorf vials of $1.5 \mathrm{~mL}$ and washed with $1 \mathrm{~mL}$ of PBSTween solution. Beads were then immobilized on a magnet and washed 3 times with $500 \mu \mathrm{L}$ of PBS-Tween. Beads were then resuspended in $40 \mu \mathrm{L}$ of biotinylated $\mathrm{hIgG}$ solution at $0.5 \mathrm{mg} / \mathrm{mL}$ and $160 \mu \mathrm{L}$ of PBS. Vials were then incubated at $25^{\circ} \mathrm{C}, 450 \mathrm{rpm}$ for $30 \mathrm{~min}$ on a Thermomixer, and beads were immobilized on a magnet and supernatants are collected and kept at $4{ }^{\circ} \mathrm{C}$. Beads were washed 4 times with $500 \mu \mathrm{L}$ of a solution of PBS-Tween. Washing solutions were kept at $4{ }^{\circ} \mathrm{C}$.

Protein G magnetic beads One hundred microliters of Pierce Protein $\mathrm{G}$ Magnetic Beads $(10 \mathrm{mg} / \mathrm{mL})$ was added to 4 LoBind Eppendorf vials of $1.5 \mathrm{~mL}$ and washed with $500 \mu \mathrm{L}$ of Tris-Tween solution and gently vortexed. The process was repeated one time with $1 \mathrm{~mL}$ of Tris-Tween. Beads were resuspended in $217 \mu \mathrm{L}$ of human IgG solution at $0.23 \mathrm{mg} / \mathrm{mL}$ with $283 \mu \mathrm{L}$ of Tris-Tween. Vials were then incubated at $25{ }^{\circ} \mathrm{C}, 650 \mathrm{rpm}$ for $1 \mathrm{~h}$ on a Thermomixer, and beads were immobilized on a magnet and supernatants were collected and kept in the fridge for analysis. Beads were then washed 2 times with $500 \mu \mathrm{L}$ with Tris-Tween. Washing solutions were kept in the fridge for analysis.

\section{Characterization of the hlgG grafting}

Enzymatic digestion of grafted antibodies For all digestions, $2 \mu \mathrm{L}$ aliquot of $100 \mathrm{U}$ of IdeS FabRICATOR enzyme and $50 \mu \mathrm{L}$ of phosphate buffer (100 mM, pH 7.0) were added to the sample, hIgG solution $(60 \mu \mathrm{L})$, or hIgG grafted on magnetic beads (suspended) and incubated for $1 \mathrm{~h} 30$ at $37{ }^{\circ} \mathrm{C}$, $300 \mathrm{rpm}$ on a thermomixer. Bead suspensions were then placed on a magnet, and supernatants were collected and analyzed.

Influence of bead grafting conditions on FLD signal for hlgG, $\mathrm{F}(\mathrm{ab})_{2}$, and $\mathrm{Fc}$ fragments To avoid any bias in the measurements, the influence of bead grafting protocols (medium, incubation steps) on hIgG FLD signals was investigated. Each sample was prepared in duplicate. hIgG samples at $0.1 \mathrm{mg} / \mathrm{mL}$ were incubated following conjugation protocols corresponding to tosylactivated carboxylic and protein $\mathrm{G}$ beads and then analyzed by SEC-FLD. 


\section{Calibration curves}

hlgG Calibration curve for whole hIgG was obtained with $0.1 \mathrm{mg} / \mathrm{mL} \mathrm{hIgG}$ solution in PBS. Dilution series of $\mathrm{hIgG}$, ranging from 0.05 to $0.7 \mu \mathrm{g}$, have been analyzed to establish the calibration curve.

hlgG fragments Calibration samples were made from IgG solution submitted to the different conjugation's protocols. hIgG solutions $(0.23 \mathrm{mg} / \mathrm{mL})$ were digested with $100 \mathrm{U}$ of IdeS and analyzed by SEC-FLD. Volumes of hIgG digests from 0.5 to $7 \mu \mathrm{L}$ were analyzed, and a calibration curve per conjugation protocol has been built for the two fragments $\left(\mathrm{F}(\mathrm{ab})_{2}\right.$ and $\left.\mathrm{Fc}\right)$.

\section{Quantitation of non-grafted hlgG in the supernatant}

Antibodies remaining in the supernatant after the grafting step were first quantified. Then, washing solutions were concentrated by ultrafiltration on $100 \mathrm{kDa}$ membrane. Five hundred microliters of washing solution was added to the filter cap and centrifuge for $4 \mathrm{~min}$ at $10,000 \mathrm{rpm}$ at $4{ }^{\circ} \mathrm{C}$ on a Centrifuged MicroStar 17R (VWR). The process was repeated until all the solution was filtered. Retentate volumes were measured before SEC-FLD analysis.

\section{Immunocapture of TNF-a by bio-functionalized magnetic beads}

One milligram of $\mathrm{COOH}$, Ts, and protein $\mathrm{G}$ magnetic beads was grafted with anti-human-TNF- $\alpha$ monoclonal antibodies from the Invitrogen ELISA kit for human TNF- $\alpha$ following the procedure described previously. Five replicates of $100 \mu \mathrm{g}$ of grafted magnetic beads of each type were then incubated for $4 \mathrm{~h}$ at $25^{\circ} \mathrm{C}$ with $5 \mathrm{ng}$ of human TNF- $\alpha$ in $250 \mu \mathrm{L}$ of PBS $(1 \times)$. Three blank samples were also prepared using grafted magnetic beads and PBS $(1 \times)$. Beads were washed with $500 \mu \mathrm{L}$ of PBS-Tween for $10 \mathrm{~min}$ at $800 \mathrm{rpm}$ on a Thermomixer. Then, heated $95{ }^{\circ} \mathrm{C}$ for $5 \mathrm{~min}$ in $250 \mu \mathrm{L}$ of PBS $(1 \times)$ for thermal elution of TNF- $\alpha$. Eluates were diluted on PBS $(1 \times, 10 \times, 100 \times)$ to match immunoassay detection range. Human TNF- $\alpha$ contents of non-diluted and diluted eluates were quantified using Invitrogen ELISA kit for human TNF- $\alpha$ using duplicates. Calibration curves were built by preparing serial dilution of human TNF- $\alpha$ solution at $500 \mathrm{pg} / \mathrm{mL}$ with and without thermal treatment at $95{ }^{\circ} \mathrm{C}$ for $5 \mathrm{~min}$ to take into account the thermal effect on TNF- $\alpha$.

\section{Results and discussion}

The first part of the study was dedicated to the development of an analytical method to evaluate the grafting efficiency on four types of beads. Two main criteria have been considered: number of antibodies grafted on the bead's surface and their orientation. For this purpose, we specifically cleaved hIgG grafted on the bead surface under the hinge region with an enzyme, IdeS, and analyzed the digestion products by SECFLD analysis. If the hIgG is immobilized via its Fc region, the $\mathrm{F}(\mathrm{ab})_{2}$ fragment will be released in the supernatant, whereas for the immobilization through $\mathrm{F}(\mathrm{ab})_{2}$ fragment, Fc fragment will be obtained.

\section{Influence of the grafting conditions on native and digested human IgG fluorescent detection}

We first developed a SEC-fluorescent detection (FLD) method to quantify hIgG in solution. Analysis of human $\operatorname{IgG}$ in solution $(0.1 \mathrm{mg} / \mathrm{mL})$ showed two peaks (Fig. 1A): the first one corresponds to a dimeric form of hIgG $(\sim 8.2 \mathrm{~min})$ and the second to the monomeric hIgG ( $9.2 \mathrm{~min})$. As the first peak area was very small compared to the 2 nd one (peak area ratio: $\sim 8$ ), dimeric hIgG was not considered for hIgG calibration. Repeatability and intermediate precision of SEC-FLD method have been evaluated. The intra- $(n=3)$ and inter-day $(n=3)$ RSD of the retention times (Tr) were less than $0.1 \%$. Resolution and limit of quantification were also evaluated at 1.3 and $160 \mathrm{nM}$ respectively (see Supplementary Material, method validation chapter).

Considering the antibody grafting density, it is important to note that for each type of beads, the grafting protocol differs in terms of buffers (i.e., $\mathrm{pH}$ and ionic strength) thermal and/or agitation that may impact protein conformation and thereby fluorescent intensities measured. Therefore, $\mathrm{hIgG}$ free in solution $(0.1 \mathrm{mg} / \mathrm{mL})$ were submitted to experimental conditions mimicking the grafting protocols applied to $\mathrm{COOH}$ and Ts beads. The agitation used for $\mathrm{hIgG}$ grafting on $\mathrm{COOH}$ beads (rotating wheel at $4{ }^{\circ} \mathrm{C}$ overnight) led to a slight decrease of the FLD signal ($5.2 \%$ ) compared to the reference sample of hIgG (PBS at $4{ }^{\circ} \mathrm{C}$ ). The FLD signal further decrease (19\%) when EDC and SulfoNHS were added in the reaction medium. To explain this decrease, we hypothesized that the temperature $\left(4^{\circ} \mathrm{C}\right)$ as well as EDC and Sulfo-NHS could increase the intra-amino acid interactions favoring thereby conformation where fluorescent amino acids are hindered. For Ts beads, an increase of the FLD signal $(+9.6 \%)$ was observed with the Ts grafting protocol (stirring at $37{ }^{\circ} \mathrm{C}$ during $16 \mathrm{~h}$ ). This signal is increased by $18 \%$ when Ts conjugation protocol is performed in borate buffer $\mathrm{pH} 9.5$ (Fig. 1B). The increase of the fluorescent signal could be related to a partial denaturation of the hIgG. Several studies have demonstrated that thermally induced unfolding of proteins led to the exposure of the fluorescent amino previously buried (e.g., tryptophan) increasing thereby the fluorescent signal [26]. The same type of experiments has been performed for hIgG cleaved by IdeS. Digested $\operatorname{IgG}(0.22 \mathrm{mg} / \mathrm{mL})$ has been first analyzed with SEC-FLD, showing two peaks: 1st peak ( $9.99 \mathrm{~min})$ 


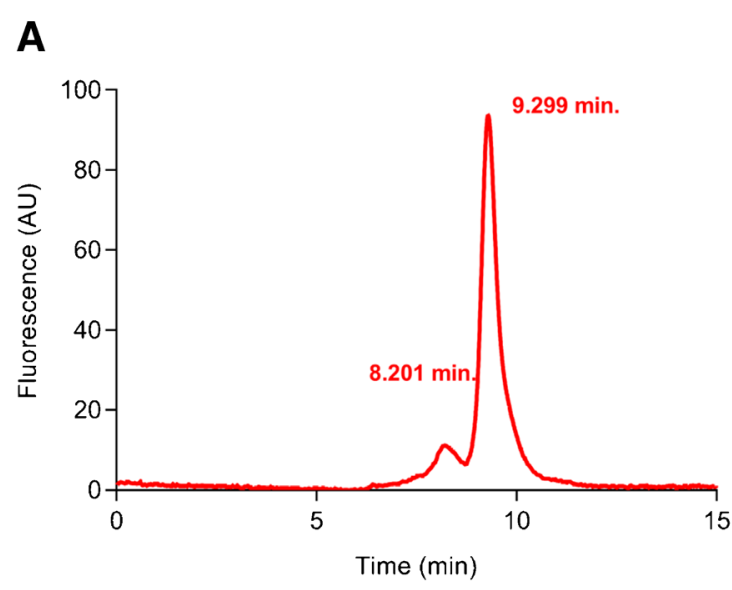

Fig. 1 Analysis of $\operatorname{hIgG}(0.1 \mathrm{mg} / \mathrm{mL})$ by SEC-FLD. A control sample (human IgG in PBS at $4{ }^{\circ} \mathrm{C}$ ). B Influence of bead grafting conditions on FLD signal of human IgG. COOH conditions are agitation at $20 \mathrm{rpm}$ on rotating wheel at $4{ }^{\circ} \mathrm{C}$ overnight in PBS, with $10 \mathrm{mg} / \mathrm{mL}$ of EDC and Sulfo-NHS. Ts conditions are agitation a $650 \mathrm{rpm}$ in a Thermomixer at

corresponding to $\mathrm{Fab}_{2}$ fragment of $100 \mathrm{kDa}, 2$ nd peak ( $10.98 \mathrm{~min}$ ) to $\mathrm{Fc}$ one $(50 \mathrm{kDa})$ (Fig. 2). Four calibration curves of hIgG cleaved by IdeS into $\mathrm{Fab}_{2}(\sim 100 \mathrm{kDa})$ and $\mathrm{Fc}(\sim$ $50 \mathrm{kDa}$ ) fragments have been performed under the grafting conditions selected for the 4 types of beads (Fig. 3). A variation has been observed for Fc peaks: a 25\% decrease of the curve's slope for $\mathrm{COOH}$ bead conditions compared to Ts beads which could lead to underestimate an unfavorable orientation (see Fig. 3A and B). Therefore, we can conclude from these results that calibration curves of hIgG and its fragments have to be established for each type of beads studied (i.e., $\mathrm{COOH}$, Ts, streptavidin, protein $\mathrm{G}$ ) to evaluate accurately the antibody grafting density and orientation.

\section{Antibody grafting density and orientation on magnetic microbeads}

We then evaluated the grafting density and $\mathrm{hIgG}$ orientation on the four types of beads: carboxylated, tosylated, protein G,

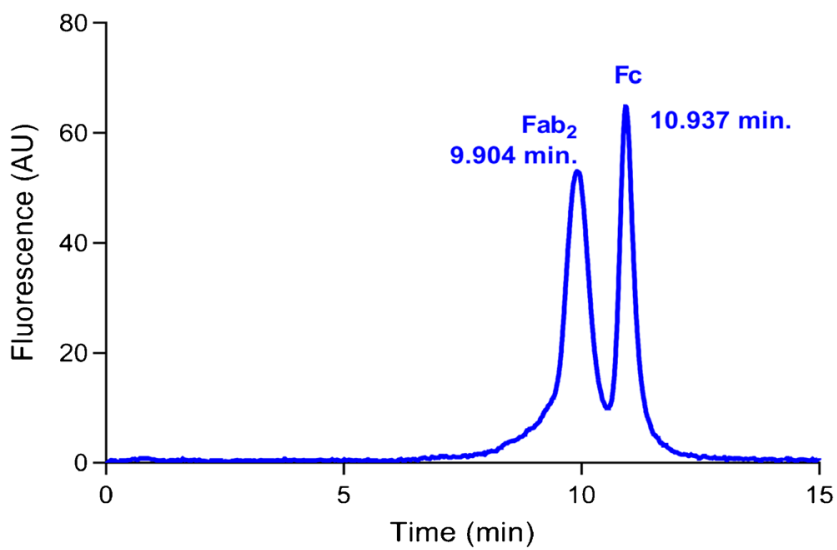

Fig. 2 SEC-FLD analysis of human IgG at $0.22 \mathrm{mg} / \mathrm{mL}$ in PBS after IdeS digestion. Experimental conditions are mentioned in Fig. 1

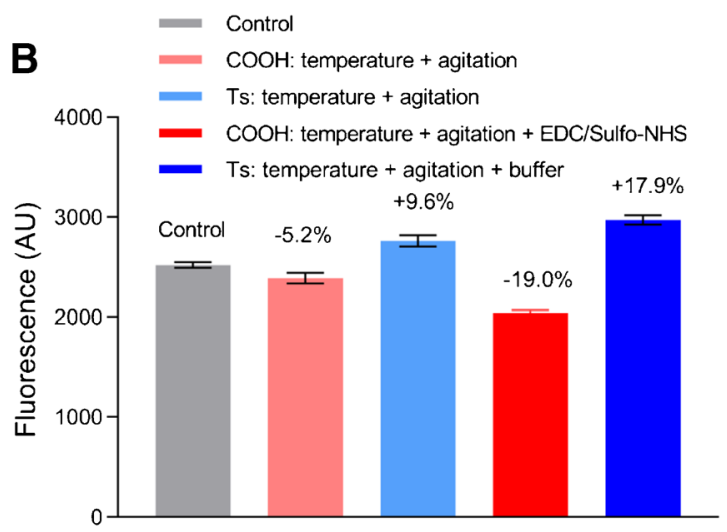

$37^{\circ} \mathrm{C}$ for $16 \mathrm{~h}$ with borate and ammonium buffer $\mathrm{pH}$ 9.5. Mobile phase was $50 \mathrm{mM}$ sodium phosphate, $150 \mathrm{mM} \mathrm{NaCl}$ and $10 \%$ isopropanol pH 6.5. Column: BioSEC-3 $(4.6 \mathrm{~mm} \times 300 \mathrm{~mm}, 300 \AA$, particle diameter $3 \mu \mathrm{m}$ ); flow rate: $300 \mu \mathrm{L} / \mathrm{min}$; FLD: $\lambda \mathrm{ex}=280 \mathrm{~nm}, \lambda \mathrm{em}=340 \mathrm{~nm}$

and streptavidin. For this purpose, magnetic beads bearing $\mathrm{hIgG}$ have been digested by IdeS and the fragments released were analyzed by SEC-FLD.

A

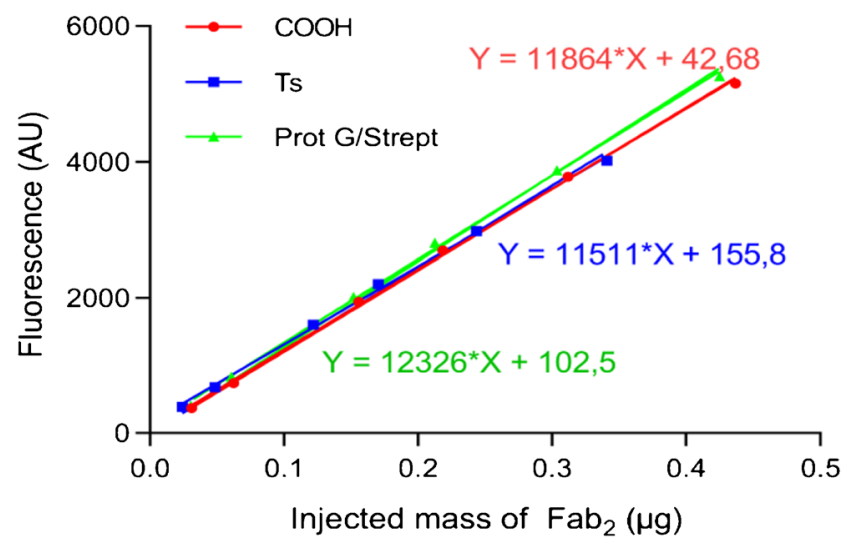

B

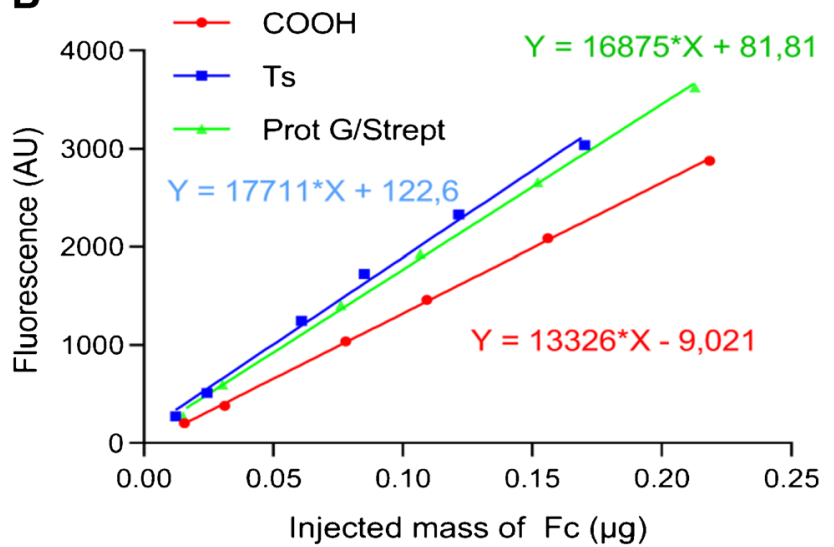

Fig. 3 Calibration curves corresponding to grafting conditions. A F(ab) fragment. B Fc fragment. Experimental conditions are mentioned in Fig. 1 


\section{$\mathrm{F}(\mathrm{ab})_{2}$ and $\mathrm{Fc}$ quantitation}

We first verified that IdeS did not overlap with peaks corresponding to hIgG fragments in our experimental conditions by analyzing $I d e S(5 \mathrm{U})$ as a control. Indeed, molecular weight of Ides is approximately $37 \mathrm{kDa}$ which might interfere with hIgG fragment detection. For IdeS, two peaks were observed at $10.08 \mathrm{~min}$ (dimer) and $11.0 \mathrm{~min}$ (monomer) with retention times close to $\mathrm{F}(\mathrm{ab})_{2}$ and $\mathrm{Fc}$ ones which could lead to overestimate peak areas related to $\mathrm{Fc}$ and, into a lesser extent, to $\mathrm{F}(\mathrm{ab})_{2}$ (see Fig. S1 in Electronic Supplementary Material). Therefore, to accurately quantify the amount of $\mathrm{F}(\mathrm{ab})_{2}$ and Fc released after the digestion of hIgG grafted on magnetic beads, a calibration curve for IdeS (from 1 to $14 \mathrm{U}$ ) was drawn using different injection volumes (see Fig. S2, Electronic Supplementary Material) and was used to evaluate IdeS contribution in $\mathrm{F}(\mathrm{ab})_{2}$ and $\mathrm{Fc}$ peak area values. Therefore, peak areas of $\mathrm{F}(\mathrm{ab})_{2}$ and $\mathrm{Fc}$ fragments were corrected with IdeS calibration in the whole study allowing a more accurate quantification (see Table S1, Supplementary material).

\section{Grafting density and hlgG orientation on the different types of beads}

To estimate the percentage of hIgG grafted (\%), washing fractions and supernatants were analyzed and Eq. 1 was used to estimate this value:

$\%_{\text {grafted }}=\frac{m_{\mathrm{i}}-\left(m_{\text {washing }}+m_{\text {supernatant }}\right)}{m_{\mathrm{i}}}$

where $m_{\mathrm{i}}$ is the initial quantity of hIgG per milligrams of beads before grafting, $m_{\text {washing }}$ and $m_{\text {supernatant }}$ are the quantity of $\mathrm{hIgG}$ found after the grafting step in washing solutions and supernatants, respectively. A calibration curve of nondigested hIgG in solution in PBS was performed to quantify remaining hIgG in the supernatant and washing solution (see Fig. S2 in Supplementary Material).

From the percentage values evaluated for the different beads (Fig. 4A), we can conclude that protein $\mathrm{G}$ beads can graft more IgG than other beads $(95.1 \%$ of injected IgG are grafted), with $\mathrm{COOH}$ beads being the less efficient with a lower density of IgG grafted on their surface (73.8\%).

The number of $\mathrm{hIgG}$ per bead has been also evaluated by considering the average mass of an $\mathrm{hIgG}(150 \mathrm{kDa})$, the bead's density $\left(1.8 \mathrm{~g} / \mathrm{cm}^{3}\right.$ for $\mathrm{Ts}, \mathrm{COOH}$, and streptavidin beads, $2.0 \mathrm{~g} / \mathrm{cm}^{3}$ for protein $\mathrm{G}$ beads), and average diameter of the beads $(1 \mu \mathrm{m})$, using the following equation:

Number of IgG molecules per bead

$$
=\frac{\%_{\text {grafted }} \times m_{\mathrm{i}}}{m_{\mathrm{IgG}}} \times \frac{m_{\text {bead }}}{1 m \mathrm{~g} \text { of beads }}
$$

where $\%_{\text {grafted }}$ is the proportion of grafted $\mathrm{hIgG}$ on the beads, $m_{\mathrm{i}}$ the initial mass $(\mu \mathrm{g})$ of hIgG incubated with $1 \mathrm{mg}$ of beads for grafting, $m_{\mathrm{IgG}}$ the mass $(\mu \mathrm{g})$ of one $\mathrm{hIgG}$ molecule, $m_{\text {bead }}$ the mass $(\mu \mathrm{g})$ of the corresponding bead.

According to Fig. 4B, protein $\mathrm{G}$ beads grafted a high number of hIgG ( 70,000 molecules per bead), followed by Ts beads ( 56,000 IgG molecules per bead), streptavidin beads $(\sim 25,000 \mathrm{IgG}$ molecules per beads) and finally $\mathrm{COOH}$ ones ( 12,000 IgG molecules per beads). We can note that despite a higher quantity of hIgG employed for carboxylic beads $(79 \mu \mathrm{g})$ compared to protein $\mathrm{G}(49.91 \mu \mathrm{g})$, the amount of $\mathrm{hIgG}$ grafted is better for protein $\mathrm{G}$ beads. The same observation can be made for Ts beads, where better grafting compared to carboxylic beads is observed despite the smaller concentration of hIgG used $(39.6 \mu \mathrm{g})$. These results suggest that the grafting efficiency is not only related to the amount of $\mathrm{hIgG}$ in the immobilization solution but also to the surface chemistry.

The hIgG orientation has been evaluated by IdeS digestion and the analysis of released fragments. Figure $5 \mathrm{~A}$ displays the different chromatograms obtained for $\mathrm{COOH}$, Ts, streptavidin, and protein $\mathrm{G}$ beads. The peak areas and notably $\mathrm{F}(\mathrm{ab})_{2} / \mathrm{Fc}$ ratio highlighted the differences between beads in terms of number of hIgG grafted and hIgG orientations. From Fig. 5A, we have observed that protein $\mathrm{G}$ beads lead to a larger peak of $\mathrm{F}(\mathrm{ab})_{2}$ compared to other beads. The orientation ratio $\left(\mathrm{F}(\mathrm{ab})_{2} /\left(\mathrm{F}(\mathrm{ab})_{2}+\mathrm{Fc}\right)\right.$ showed that protein $\mathrm{G}$ beads also exhibit the best orientation $(\sim 73.8 \%$ of antibodies grafted by their $\mathrm{Fc}$ fragment) (Fig. 5B) compared to Ts (31.3\%), streptavidin (9.9\%), and carboxylic beads (5.2\%). We can conclude from these results that protein $\mathrm{G}$ led not only to more grafted antibodies but also to a higher proportion of well orientated immobilized hIgG. This could be due to the surface chemistry of carboxylic and Ts beads that led to a random immobilization of hIgG via amino (carboxylic and Ts) and sulfhydryl groups (Ts). Therefore, only a fraction of the $\mathrm{F}(\mathrm{ab})_{2}$ binding sites will be potentially available for biorecognition. The better orientation ratio observed for Ts compared to carboxylic beads could be related to the hydrophobic surface of tosyl beads favoring hIgG orientation through the more hydrophobic $\mathrm{Fc}$ region. In contrast, for protein $\mathrm{G}$, immobilization through $\mathrm{Fc}$ fragment allows for a better orientation with a higher proportion of $\mathrm{F}(\mathrm{ab})_{2}$ available.

\section{IdeS digestion efficiency on the magnetic beads}

To ensure that the better peak area ratio $\mathrm{F}(\mathrm{ab})_{2} / \mathrm{Fc}$ observed in protein $\mathrm{G}$ was related to a better orientation and not to a higher enzymatic digestion efficiency, IdeS digestion yield has been estimated (Eq. 3).

$\%_{\text {digested }}=\frac{m_{\text {digested }}}{m_{\mathrm{i}}-\left(m_{\text {washing }}+m_{\text {supernatant }}\right)}$ 
A

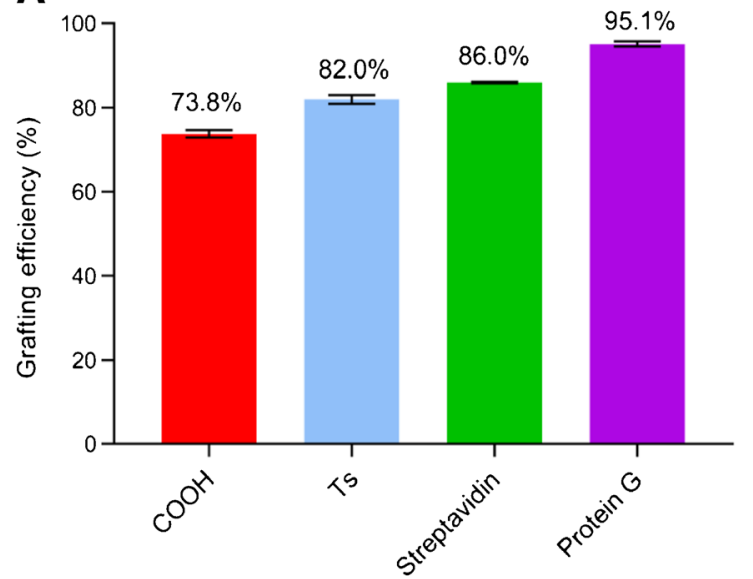

B

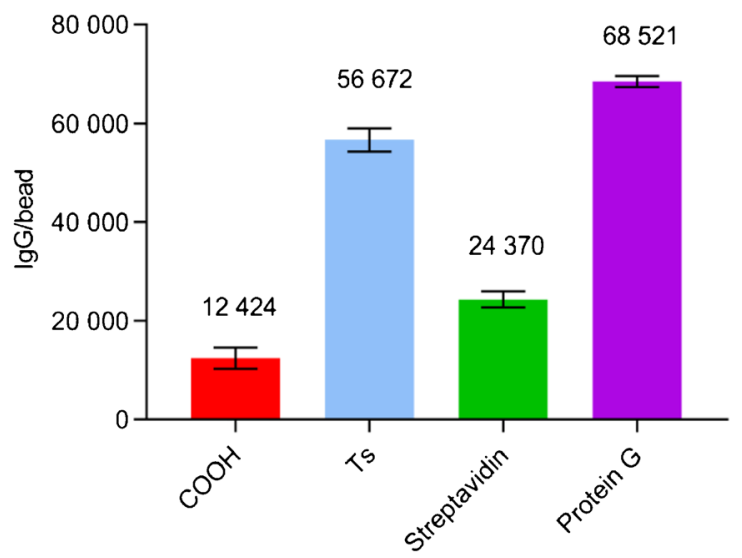

Fig. 4 hIgG immobilization study on beads. A Grafting efficiency. B number of hIgG molecules per bead

Digestion efficiency $\%_{\text {digested }}$ introduced in Eq. 3 is directly correlated to biologically available hIgG, where $m_{\mathrm{i}}$ is the initial quantity of hIgG per milligrams of beads before grafting, $m_{\text {washing }}$ and $m_{\text {supernatant }}$ are the quantity of hIgG found in washing liquids and supernatants, and $m_{\text {digested }}$ is the quantity of hIgG found in the digestate after IdeS digestion.

Indeed, we considered that IdeS cannot fully digest every hIgG molecule grafted on the bead, which means that this method could give an underestimated quantification of hIgG available to IdeS molecules. Being a relatively large molecule $(\sim 37 \mathrm{kDa})$, IdeS may not access easily to the hinge region of some hIgG molecules within the antibody layers (or multilayers), resulting in a partial proteolysis yield. Assuming that these unavailable $\mathrm{hIgG}$ molecules would not be available for other reactions with biomolecules (such as antigen recognition and capture), we can suppose that the digestion yield also reflects the antigen capture potential of the beads. The results showed that IdeS digestion yield is higher for tosylactivated beads $(46.3 \%)$ than protein $\mathrm{G}(34.2 \%)$ and streptavidin $(37.5 \%)$. Unexpectedly, we found a very low digestion yield for $\mathrm{COOH}$ beads (5.6\%), as depicted in Fig. 6 .

The lower digestion yield for protein $\mathrm{G}$ beads compared to the tosylactivated beads is probably related to the higher grafting density leading thereby to a steric hindrance detrimental for IdeS biological activity. Considering the better ratio $\mathrm{F}(\mathrm{ab})_{2} /\left(\mathrm{F}(\mathrm{ab})_{2}+\mathrm{Fc}\right)$ and the lower digestion yield of IdeS for protein $\mathrm{G}$ beads, these results confirm the better orientation of hIgG on this kind of beads.

Therefore, the overall results showed that protein $\mathrm{G}$ beads exhibit the best orientation ratio as well as the best grafting density. This could be explained by their ability to capture hIgG through $\mathrm{Fc}$ fragment, leading thereby to a more favorable orientation [27]. In contrast, hIgG are randomly immobilized by covalent bonding for $\mathrm{COOH}$ and Ts beads and via biotin interactions (non-covalent) for streptavidin beads [28]. In addition, streptavidin beads rely on a biotinylation procedure prior to antibody grafting on beads. This additional step may decrease the efficiency of the whole conjugation procedure as biotinylation will not be total. Finally, protein $\mathrm{G}$ beads offer the best results in terms of antibody orientation and density on the surface. Moreover, they are particularly easy to graft with an average of an hour and a half to complete the whole procedure compared to $18 \mathrm{~h}$ for Ts and $30 \mathrm{~h}$ for $\mathrm{COOH}$ beads, which makes them the best candidate for antibody grafting and immunocapture.

Concerning ProtA/ProtG beads, these results are in agreement with Shen et al. [17] in terms of antibody coverage and orientation ratio. In contrast, for carboxylated beads, a high difference is observed with our study. This discrepancy could be related to the digestion tool employed. Indeed, with trypsin digestion, employed by Shen et al., the peptides released from the $\mathrm{F}(\mathrm{ab})_{2}$ region, detected and quantified by LC-MS, could arise from antibodies attached on beads via one Fab fragment. This could lead therefore to an overestimation of the orientation ratio for $\mathrm{COOH}$ beads where antibodies are randomly immobilized. On Prot G/A beads, antibodies are immobilized via Fc fragments and thereby well oriented. Our method combining IdeS digestion with SEC analysis offers size discrimination, making it possible to retrieve more information to identify the fragment released (i.e., Fc $(50 \mathrm{kDa})$ vs $\mathrm{F}(\mathrm{ab})_{2}$ (100 kDa).

We have therefore developed a direct accurate and rapid quantification method of bioavailable $\mathrm{hIgG}$ on magnetic beads. Our study takes into account bead grafting conditions and IdeS contribution effect on FLD signal and absorption on beads for a more accurate quantification. Finally, we compared the performance of $\mathrm{COOH}$, Ts, and protein $\mathrm{G}$ beads for capturing TNF- $\alpha$, a cytokine involved in major depressive disorders (MDD) and other inflammatory-related pathologies. Streptavidin beads were not considered in the study as they did not provide added value compared to carboxylated and tosylactivated beads. 
A
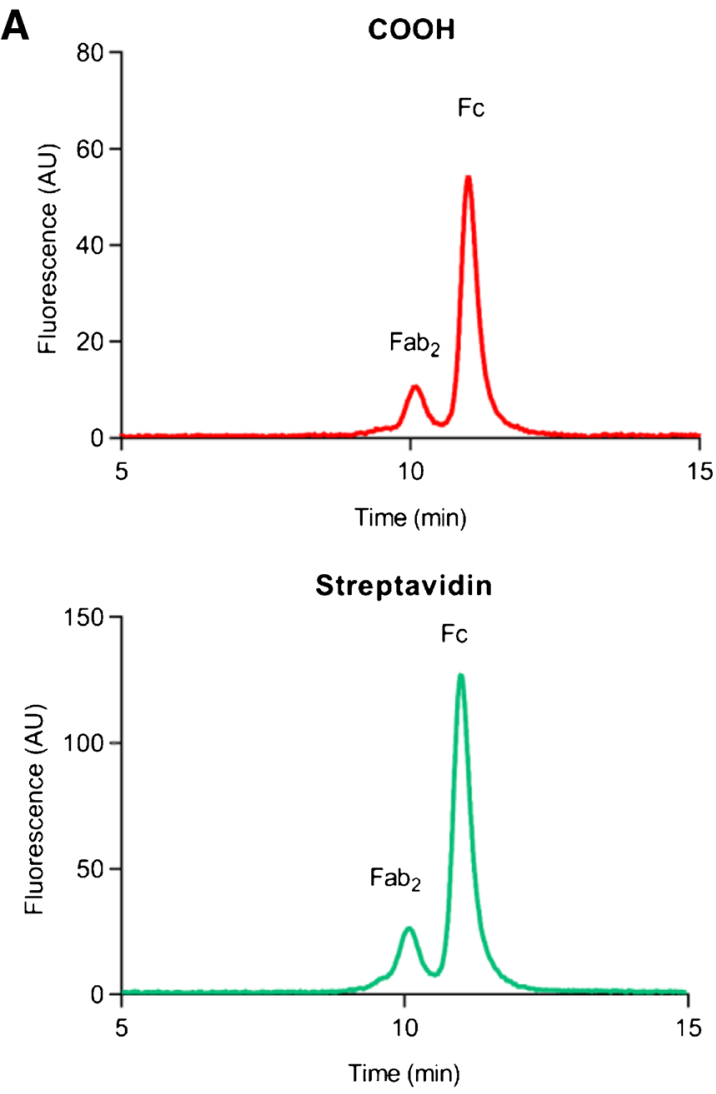

B

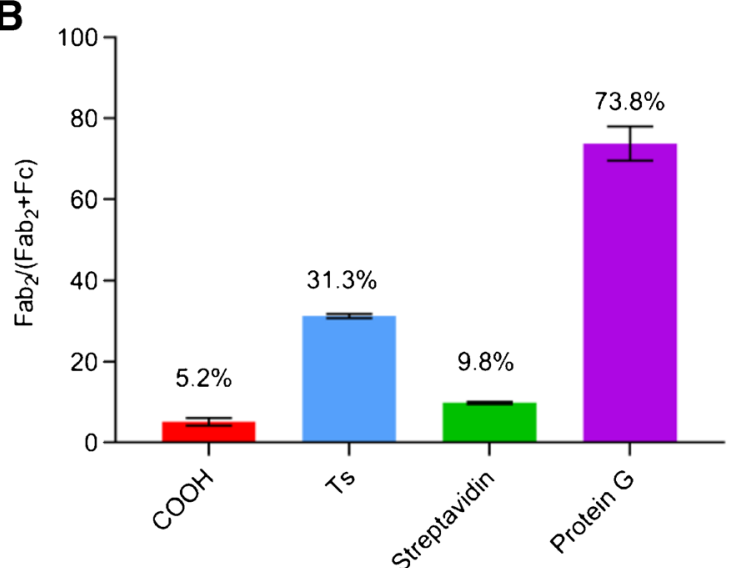

Ts

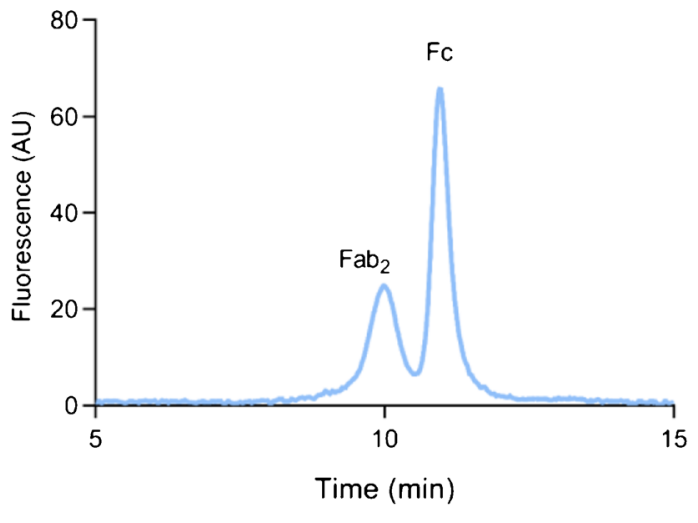

Protein G

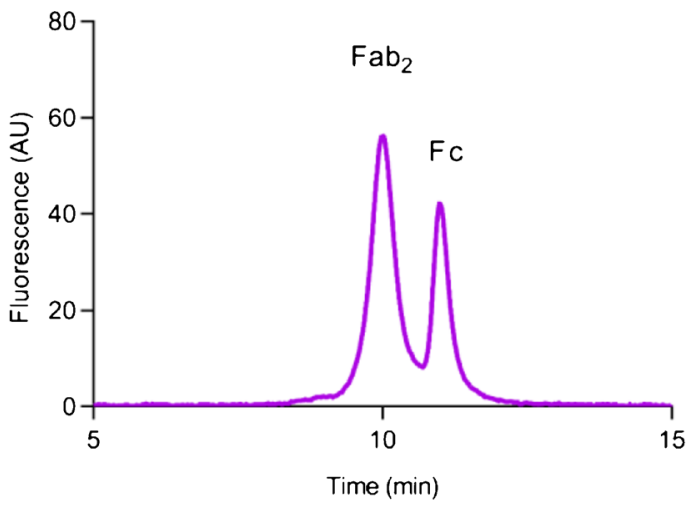

Fig. 5 IdeS digestion of immobilized hIgG. A SEC-FLD analysis. B Evaluation of the percentage of hIgG orientated with $\mathrm{F}(\mathrm{ab})_{2}$ free on the bead surface. Experimental conditions as Fig. 1

\section{Application for immunocapture of TNF-a: towards quantification of biomarkers in biological fluids}

To illustrate the performance of the previously studied magnetic beads, $\mathrm{COOH}$, Ts, and protein $\mathrm{G}$ beads were grafted with monoclonal anti-human-TNF- $\alpha$ antibodies following the procedure previously described. Five nanograms of human TNF- $\alpha$ were then incubated for $4 \mathrm{~h}$ at $25^{\circ} \mathrm{C}$ with $100 \mu \mathrm{g}$ of grafted magnetic beads of each type. Elution of antigens was done using thermal elution in $\mathrm{PBS}$ at $95^{\circ} \mathrm{C}$ for $5 \mathrm{~min}$ in order to maximize the amount of antigen release and minimize protein denaturation [29]. Eluates were then prepared in three dilutions: non-diluted, ten and one hundredfold diluted to ensure that recovered antigen concentration matched the immunoassay's linear range. Quantification was carried out using an ELISA kit for human TNF- $\alpha$ on microplate by absorbance spectrometry. A calibration curve was built using human TNF- $\alpha$ standard solution. The standard was also treated at $95{ }^{\circ} \mathrm{C}$ for $5 \mathrm{~min}$ in order to reflect possible conformational changes of TNF- $\alpha$ that would affect immunoassay detection. 


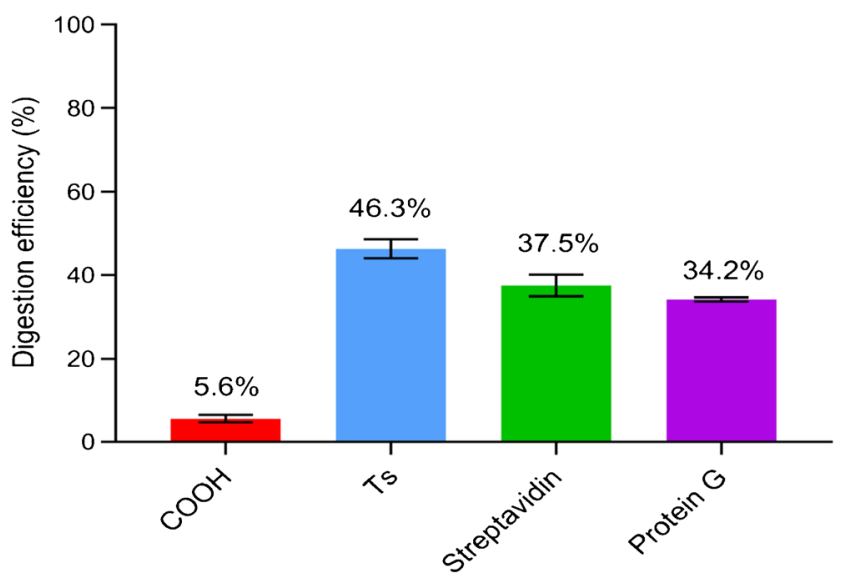

Fig. 6 Comparison of IdeS digestion efficiency for $\mathrm{COOH}$, Ts, streptavidin, and protein $\mathrm{G}$ magnetic beads

As shown in Fig. S3, thermal treatment influenced calibration curve slope with a $40 \%$ decrease in slope after thermal treatment. Quantification of eluates from the beads was therefore performed using the thermal treatment calibration curve. Results are shown in Table 1.

For $5.0 \mathrm{ng}$ of human TNF- $\alpha$ incubated with $100 \mu \mathrm{g}$ of beads, $67.6 \%$ of TNF- $\alpha$ was recovered with protein $\mathrm{G}$ beads, but only $6.6 \%$ and $2.8 \%$ for $\mathrm{COOH}$ and Ts beads, respectively. These results support the high antibody grafting efficiency of protein $\mathrm{G}$ beads in terms of both antibody coverage and orientation. For $\mathrm{COOH}$ and Ts beads, they suggest a very low capture ability of these beads for this application probably due to poor orientation and/or antibody density. However, it should be noted that thermal elution does not necessarily elute all the captured antigens. Nevertheless, it is still the mildest procedure for antigen elution without causing full protein denaturation (from acidic media) that would render accurate quantification with ELISA impossible. These results are in agreement with our SEC-FLD study highlighting the better grafting and orientation of $\mathrm{hIgG}$ on protein $\mathrm{G}$ magnetic beads. They are therefore promising candidates for sensitive and specific detection of biomarkers like TNF- $\alpha$ and could be extended to other cytokines or proteins.

\section{Conclusion}

In the present study, we present an original analytical method for the evaluation of human IgG grafting efficiency on 4 types of commercial magnetic beads for immunocapture applications. This method relies on an innovative combination of a highly specific digestion of hIgG by IdeS together with the high resolution of the fragments' analysis by SEC, offering an efficient determination of antibody orientation. This work offers a comprehensive approach of the influence of grafting conditions, IdeS, and bead's surface chemistry on fluorescent signal detection. All results support the high efficiency of protein $\mathrm{G}$ magnetic beads for a high grafting yield of human $\operatorname{IgG}(\sim 70,000 \mathrm{IgG}$ molecules per bead $)$ and an optimal orientation of antibodies for antigen capture $\left(75 \%\right.$ of $\mathrm{F}(\mathrm{ab})_{2}$ fragment free). Moreover, protein $\mathrm{G}$ beads ease-of-use in terms of grafting conditions and time make them a powerful and convenient tool for biosensing and lab-on-a-chip applications. These findings have been illustrated with a high-value application for the immunocapture and elution of human TNF- $\alpha$ at $20 \mathrm{ng} / \mathrm{mL}$, towards the monitoring of inflammatory-related pathologies. This work will be extended to other cytokines. Finally, this method can be more generally used to assess the hIgG coverage and orientation on any type of surface, relying on the high specificity and efficiency of the IdeS digestion of hIgG.

Abbreviations $\mathrm{COOH}$, Carboxyl; $E D C, \mathrm{~N}-(3-$ Dimethylaminopropyl)-N '-ethylcarbodiimide hydrochloride; $F L D$, Fluorescent detection; $h \operatorname{Ig} G$, Human immunoglobulin G; $P B S$, Phosphate-buffered saline; SEC, Size exclusion chromatography; Sulfo-NHS, N-Hydroxysulfosuccinimide sodium salt; $T B S$, Tris-buffered saline; $T S$, Tosyl

Supplementary Information The online version contains supplementary material available at https://doi.org/10.1007/s00216-021-03608-w.

Code availability Not applicable.

Author contribution Etienne Laborie: investigation, writing original draft, validation; Victor Le-Minh: investigation, writing-review; Thanh Duc Mai: writing original draft; Mehdi Ammar: resources, supervision; Myriam Taverna: resources, writing-review; Claire Smadja: funding, project supervision, writing - original draft.

Funding We thank the "Investissement d'Avenir" program, through the "IDI 2017" project funded by the IDEX Paris-Saclay, ANR-11-IDEX0003-02, which provided the financial support of Etienne Laborie as a fellowship, LabeX LaSIPS (ANR-10-LABX-0040-LaSIPS) and LabeX NanoSaclay, (ANR-10-LABX-0035).

We thank the Doctoral school of Chemical Sciences of University Paris Saclay which provided the financial support of Victor Le-Minh as a fellowship.

Data availability Not applicable.
Table 1 Comparison of bead efficiency for immunocapture of TNF- $\alpha$ ( $n=5$ replicates per bead $)$

\begin{tabular}{llll}
\hline & $\begin{array}{l}\text { COOH } \\
\text { beads }\end{array}$ & Ts beads & $\begin{array}{l}\text { Protein G } \\
\text { beads }\end{array}$ \\
\hline $\begin{array}{c}\text { TNF- } \alpha \text { recovered after thermal elution }(\% \text { RSD }) \\
(n g / 100 \mu \mathrm{g} \text { of beads) }\end{array}$ & $0.33(15 \%)$ & $0.14(13 \%)$ & $3.38(17 \%)$ \\
\hline
\end{tabular}




\section{Declarations}

Competing interests The authors declare no competing interests.

\section{References}

1. Hou Z, Liu Y, Xu J, Zhu J. Surface engineering of magnetic iron oxide nanoparticles by polymer grafting: synthesis progress and biomedical applications. Nanoscale. 2020;12:14957-75.

2. Kruszewska J, Zajda J, Matczuk M. How to effectively prepare a sample for bottom-up proteomic analysis of nanoparticle protein corona? A critical review. Talanta. 2021;226:122153.

3. Khizar S, Ben Halima H, Ahmad NM, Zine N, Errachid A, Elaissari A. Magnetic nanoparticles in microfluidic and sensing: from transport to detection. Electrophoresis. 2020;41:1206-24.

4. Liu S, Haller E, Horak J, Brandstetter M, Heuser T, Lämmerhofer M. Protein A- and protein G-gold nanoparticle bioconjugates as nano-immunoaffinity platform for human $\mathrm{IgG}$ depletion in plasma and antibody extraction from cell culture supernatant. Talanta. 2019;194:664-72. https://doi.org/10.1016/j.talanta.2018.10.079.

5. Zhang L, Hu D, Salmain M, Liedberg B, Boujday S. Direct quantification of surface coverage of antibody in IgG-gold nanoparticles conjugates. Talanta. 2019;204:875-81. https://doi.org/10.1016/j. talanta.2019.05.104

6. Kozlowski R, Ragupathi A, Dyer RB. Characterizing the surface coverage of protein-gold nanoparticle bioconjugates. Bioconjug Chem. 2018;29:2691-700. https://doi.org/10.1021/acs. bioconjchem. 8 b00366.

7. Walkey CD, Olsen JB, Guo H, Emili A, Chan WCW. Nanoparticle size and surface chemistry determine serum protein adsorption and macrophage uptake. J Am Chem Soc. 2012;134:2139-47. https:// doi.org/10.1021/ja2084338

8. Hinterwirth H, Lindner W, Lämmerhofer M. Bioconjugation of trypsin onto gold nanoparticles: effect of surface chemistry on bioactivity. Anal Chim Acta. 2012;733:90-7. https://doi.org/10.1016/ j.aca.2012.04.036.

9. Young MB, Oh BK, Lee W, Won HL, Choi JW. Study on orientation of immunoglobulin $G$ on protein $G$ layer. Biosens Bioelectron. 2005;21:103-10. https://doi.org/10.1016/j.bios.2004. 09.003 .

10. Song HY, Zhou X, Hobley J, Su X. Comparative study of random and oriented antibody immobilization as measured by dual polarization interferometry and surface plasmon resonance spectroscopy. Langmuir. 2012;28:997-1004. https://doi.org/10.1021/la202734f.

11. Yoshimoto K, Nishio M, Sugasawa H, Nagasaki Y. Direct observation of adsorption-induced inactivation of antibody fragments surrounded by mixed-PEG layer on a gold surface. J Am Chem Soc. 2010;132:7982-9. https://doi.org/10.1021/ja910372e.

12. Fujiwara $\mathrm{K}$, Watarai $\mathrm{H}$, Itoh $\mathrm{H}$, Nakahama E, Ogawa $\mathrm{N}$. Measurement of antibody binding to protein immobilized on gold nanoparticles by localized surface plasmon spectroscopy. Anal Bioanal Chem. 2006;386:639-44. https://doi.org/10.1007/ s00216-006-0559-2.

13. Liu S, Horak J, Höldrich M, Lämmerhofer M. Accurate and reliable quantification of the protein surface coverage on proteinfunctionalized nanoparticles. Anal Chim Acta. 2017;989:29-37. https://doi.org/10.1016/j.aca.2017.08.004.

14. Bouzas-Ramos D, Trapiella-Alfonso L, Pons K, Encinar JR, CostaFernández JM, Tsatsaris V, Gagey-Eilstein N. Controlling ligand surface density on streptavidin-magnetic particles by a simple, rapid, and reliable chemiluminescent test. Bioconjug Chem. 2018;29: 2646-53. https://doi.org/10.1021/acs.bioconjchem.8b00347.
15. Näreoja T, Ebner A, Gruber HJ, Taskinen B, Kienberger F, Hänninen PE, Hytönen VP, Hinterdorfer P, Härmä H. Kinetics of bioconjugate nanoparticle label binding in a sandwich-type immunoassay. Anal Bioanal Chem. 2014;406:493-503. https://doi.org/ 10.1007/s00216-013-7474-0.

16. Hua X, You H, Luo P, Tao Z, Chen H, Liu F, Wang M. Upconversion fluorescence immunoassay for imidaclothiz by magnetic nanoparticle separation. Anal Bioanal Chem. 2017;409: 6885-92. https://doi.org/10.1007/s00216-017-0653-7.

17. Shen M, Jiang D, De Silva PIT, Song B, Rusling JF. Restricted proteolysis and LC-MS/MS to evaluate the orientation of surfaceimmobilized antibodies. Anal Chem. 2019;91:4913-9. https://doi. org/10.1021/acs.analchem.9b01155.

18. Johansson BP, Shannon O, Björck L. IdeS: a bacterial proteolytic enzyme with therapeutic potential. PLoS One. 2008;3. https://doi. org/10.1371/journal.pone.0001692.

19. Sasso LA, Aran K, Guan Y, Ündar A, Zahn JD. Continuous monitoring of inflammation biomarkers during simulated cardiopulmonary bypass using a microfluidic immunoassay device-a pilot study. Artif Organs. 2013;37:E9-E17. https://doi.org/10.1111/aor.12021.

20. Kumar S, Tripathy S, Jyoti A, Singh SG. Recent advances in biosensors for diagnosis and detection of sepsis: a comprehensive review. Biosens Bioelectron. 2019;124-125:205-15.

21. Yndestad A, Damås JK, Øie E, Ueland T, Gullestad L, Aukrust P. Systemic inflammation in heart failure - the whys and wherefores. Heart Fail Rev. 2006;11:83-92.

22. Russell B, Moss C, George G, Santaolalla A, Cope A, Papa S, Van Hemelrijck M. Associations between immune-suppressive and stimulating drugs and novel COVID-19 - a systematic review of current evidence. Ecancermedicalscience. 2020;14:1022. https:// doi.org/10.3332/ecancer.2020.1022.

23. Mehta P, McAuley DF, Brown M, Sanchez E, Tattersall RS, Manson JJ. COVID-19: consider cytokine storm syndromes and immunosuppression. Lancet. 2020;395:1033-4.

24. Slavich GM, Irwin MR. From stress to inflammation and major depressive disorder: a social signal transduction theory of depression. Psychol Bull. 2014;140:774-815. https://doi.org/10.1037/ a0035302.

25. Cizza G, Marques AH, Eskandari F, Christie IC, Torvik S, Silverman MN, Phillips TM, Sternberg EM. Elevated neuroimmune biomarkers in sweat patches and plasma of premenopausal women with major depressive disorder in remission: the POWER study. Biol Psychiatry. 2008;64:907-11. https://doi.org/ 10.1016/j.biopsych.2008.05.035.

26. Brader ML, Estey T, Bai S, Alston RW, Lucas KK, Lantz S, Landsman P, Maloney KM. Examination of thermal unfolding and aggregation profiles of a series of developable therapeutic monoclonal antibodies. Mol Pharm. 2015;12:1005-17. https://doi. org/10.1021/mp400666b.

27. Choe W, Durgannavar TA, Chung SJ. Fc-binding ligands of immunoglobulin G: an overview of high affinity proteins and peptides. Materials (Basel). 2016;9.

28. Foddai A, Elliott CT, Grant IR. Maximizing capture efficiency and specificity of magnetic separation for mycobacterium avium subsp. paratuberculosis cells. Appl Environ Microbiol. 2010;76:7550-8. https://doi.org/10.1128/AEM.01432-10.

29. Mai TD, Pereiro I, Hiraoui M, Viovy JL, Descroix S, Taverna M, Smadja C. Magneto-immunocapture with on-bead fluorescent labeling of amyloid- $\beta$ peptides: towards a microfluidized-bed-based operation. Analyst. 2015;140:5891-900. https://doi.org/10.1039/ c5an01179e.

Publisher's note Springer Nature remains neutral with regard to jurisdictional claims in published maps and institutional affiliations. 